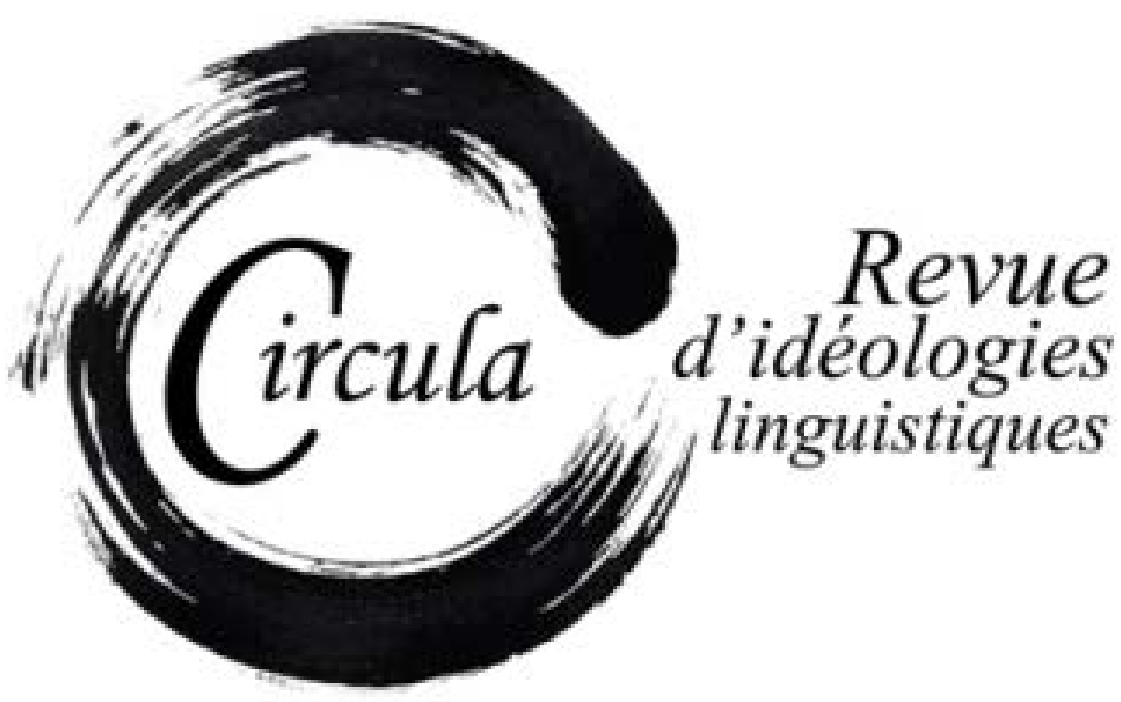

TITRE: L'IDEOLOGIA LINGUISTICA DI BRUNO MIGLIORINI GIORNALISTA

Auteur(s): Carmelo SCAVuzzo, UniversitÀ di Messina

Revue: Circula, NumÉro 2, PAgES 1-17

ISSN: 2369-6761

DiRecteurs: Wim Remysen et SABInE SchWARZE

URI: HTTP://HDL.HANDLE.NET/11143/7984

DOI: $10.17118 / 11143 / 7984$ 


\title{
L’ideologia linguistica di Bruno Migliorini giornalista
}

Carmelo Scavuzzo, Università di Messina

cscavuzzo@unime.it

\begin{abstract}
Riassunto: La ricerca indaga alcuni articoli di Bruno Migliorini apparsi nella stampa periodica e quotidiana dal 1942 al 1961. Nel rivolgere particolare attenzione alla lingua media e al suo funzionamento nella nazione, egli considera la lingua come strumento sociale e sottolinea la necessità che lo studio strutturale arricchisca la ricerca storica. Se Migliorini veste i panni del giornalista militante, mantiene sempre quelli dello storico della lingua, nella persuasione che le testimonianze storiche siano l'indispensabile ausilio a ogni studio sincronico. La ricerca fissa l'obiettivo sull'osservatorio linguistico di Migliorini e sugli elementi che suscitano il suo interesse: lingua letteraria, parole antiche e nuove, locuzioni malamente modellate, stranierismi adattati e no, voci dialettali, lingue speciali. Ne emerge l'azione educatrice di un linguista, che esce dal chiuso dell'accademia e si rivolge ai lettori dei giornali dell'epoca, affinché prendano coscienza del valore e del significato della loro lingua.
\end{abstract}

Parole chiave: giornali; storia della lingua italiana; stranierismi; lingua letteraria; lingua comune; lingue speciali

\begin{abstract}
This research investigates some articles Bruno Migliorini published in magazines and newspapers from 1942 to 1961. Migliorini pays particular attention to everyday language and its function in the country. He therefore considers language as a social tool and underlines the need for a structural study that is aimed at enriching historical research. Migliorini is both a militant journalist and a historian of language, and, as such, he is persuaded that historical evidence support synchronic studies. This research sheds light on Migliorini's linguistic observatory and on the elements that interest him: literary language, ancient and new words, badly formed phrases, adapted and not adapted foreign words, dialect words, special languages. The educational action of Migliorini, a linguist who comes out from the academic context by addressing the readers of newspapers of his time and by making them aware of the value and meaning of their language, will also emerge in this study.
\end{abstract}

Keywords: newspapers; history of Italian language; foreign words; literary language; everyday language; special languages 


\section{Introduzione}

Bruno Migliorini è stato, è ben noto, una personalità spiccata di studioso, capace d'indagare le questioni centrali della storia linguistica italiana. In questa sede rivolgerò l'attenzione alle rubriche giornalistiche in cui il linguista trasferiva con perseverante continuità qualche scampolo del suo alto magistero scientifico ${ }^{1}$. Anticipo che ho preso in esame solo articoli apparsi sulla stampa (quotidiana e periodica) e non più ripubblicati, lungo un arco cronologico che va dal 1942 al $1961^{2}$. La destinazione giornalistica e l'intento divulgativo a essa intrinseco giustificano il carattere più provvisorio di qualche pagina, ma c'è una sostanziale analogia tra la scrittura di Migliorini giornalista e quella del saggista.

È noto come le schede da lui compilate con cadenza quasi giornaliera ${ }^{3}$ si traducessero sovente in articoli accolti dalla stampa quotidiana e periodica (dalla prima più che dalla seconda): si tratti della noterella etimologica, della storia di una parola o di una semplice curiosità ortografica, ogni elemento della lingua è indagato con uguale umiltà, perché anche la ricerca «minore» in lui si alimenta stabilmente della certezza sulle prospettive che ne possono scaturire e sul filone di studi a cui rifarsi.

Sono perlopiù risposte a dubbi e lagnanze di singoli utenti che attraverso il giornale segnalano pubblicamente una trasgressione grammaticale; sono recensioni ricche di proposte e consigli; sono rubriche che non ricercano facili effetti, ma di sicura efficacia pragmatica e di gradevole vivacità, con un taglio discorsivo, sentito come il più adatto alla registrazione dei fenomeni vivi e contingenti della lingua. Codesto abito di osservazione realistico, e tuttavia incline all'ascolto e alla comprensione, si serve di un discorso nitido e privo di ambiguità; lo stile è quello di sempre: asciutto, essenziale, garbatamente arguto (sullo stile del Migliorini uomo e saggista cf. Folena, 1979 e Colussi, 2008).

\footnotetext{
1. È ben presente a Migliorini l'idea che già nel primo Novecento il giornale costituisca un importante modo della comunicazione destinato a incidere sulle sorti della nostra lingua e a coinvolgere anche «quegli individui su cui l'influenza della letteratura non arriverebbe a farsi sentire» (Migliorini, 1990: 6).

2. Questi i titoli degli articoli consultati in Settegiorni: «Autarcia», 1 agosto 1942, p. 20; «Puericoltura», 22 agosto 1942, p. 20; «Lingua nazionale e contributi dialettali», 19 settembre 1942, p. 11; «La Spezia» e «L'Aquila», 31 ottobre 1942, p. 20; «Disinfezione del vocabolario: parole per aria» [rec. P. Monelli, Barbaro dominio, 2ª ed. Milano, 1943], 27 febbraio 1943, p. 9. Della rubrica Vocabolario del Corriere della Sera ho esaminato i seguenti titoli: «Estrogeno», «Convergenze parallele» (2 agosto 1960, p. 3), «Semianalfabeti», «Cipolla» (13 settembre 1960, p. 3), «Kartismo» (11 ottobre 1960, p. 3), «Reazionario» (21 ottobre 1960, p. 3), «Glamour» (23 dicembre 1960, p. 3), «Carrellino per la spesa» (18 gennaio 1961, p. 3). Della rubrica Osservatorio linguistico della Nazione di Firenze ho analizzato questi articoli: «Grammatica e burocrazia» (11 agosto 1954, p. 3), «La metropolitana» (17 febbraio 1955, p. 3). Della rubrica Specola linguistica del Tempo ho preso in esame gli articoli che seguono: «Settennato o settennio?» (20 maggio 1948, p. 3), «Due parole di moda Succube? controproducente» (21 luglio 1948, p. 3), «Olimpico e olimpionico non hanno lo stesso senso» (19 settembre 1948, p. 3), «Più si parla di De Gaulle e più i Gollisti si rafforzano» (10 novembre 1948, p. 3). Avverto che la lista dei succitati articoli di quotidiani e periodici non figura nella bibliografia conclusiva, nella quale ho ritenuto opportuno, anche per ragioni di spazio, inserire soltanto gli studi e non le fonti. Lo stesso vale per altre fonti giornalistiche occasionalmente citate.
}

3. Nulla dies sine schedula era sentenza assai cara a Migliorini (cf. Baldelli,1979: 20). 
Accade che il motto arguto suggelli la discussione linguistica; ad esempio, parlando delle scienze che si occupano di bambini, Migliorini ironizza sull'abitudine tutta italiana di coniare parole pompose e solenni, anche per designare professioni comuni: «quel che è peggio, la puericultura minaccia anche di figliare una puericultrice» (Settegiorni, 22 agosto 1942).

Altra volta, la presenza in Gadda e Monelli di voci e costruzioni vernacole gli ispira un atteggiamento bonariamente scherzoso, promosso dal meccanismo analogico, per cui una corrente letteraria di primo Novecento, nota per le sue tendenze modernistiche, diventa il nome di una città: «essi si guadagnano il cuore di un certo numero di strapaesani, ma si fanno anche non pochi nemici a Stracittà» (Settegiorni, 19 settembre 1942): su Strapaese e Stracittà cf. Migliorini (1990: 57, 160-161). Un siffatto tono accattivante appare a Migliorini il più adatto per parlare dei consistenti apporti dialettali alla lingua comune; il romaneschismo rione, che è prevalso sulle forme concorrenti (sesti, sestieri, quarti, quartieri, terzi), «da poco ha figliato l'aggettivo rionale (mentre l'antico capo del rione, cioè il caporione, ha perduto la dieresi, ma non la riottosità)»; ridarello è stato più fortunato di altri termini locali, «forse perché è arrivato a inserirsi senza chiasso».

Di là dalla battuta spiritosa, c'è l'interesse per le varietà dialettali e per le aree semantiche più rappresentate; c'è la volontà di verificare la corrispondenza del dialettalismo di maggior vitalità con quello meno diffuso: di qui la conclusione che la voce settentrionale mezzadro, ad esempio, ha ormai sostituito l'equivalente toscano mezzaiolo (cf. anche Migliorini, 1978: 725). Le manifestazioni d'insofferenza tipiche dei puristi ottocenteschi non gli appartengono ${ }^{5}$; Migliorini evita i proclami, preferisce affidarsi al (buon) gusto del lettore di turno.

Che cosa è una lingua? Come funziona una lingua? Questi i quesiti a cui si propone di rispondere il giornalista Migliorini; oggetto di discussione diventano fatalmente sia la formazione delle lingue, sia i suoni, le forme, i vocaboli. Quella lessicale, in particolare, si sarebbe imposta anche qui come l'indagine d'elezione ${ }^{6}$. Nell'affidare ai giornali le sue riflessioni, egli si appoggia stabilmente sull'idea che le ricerche storiche, anche le più documentate ed erudite, possano essere arricchite dallo studio strutturale della lingua.

Quel che più gli sta a cuore è comunque la lingua come strumento sociale, ovvero l'italiano medio della collettività. Pure consapevole di quanto grande sia stata nei secoli l'influenza dei grammatici nella storia dell'italiano, Migliorini solo di rado prescrive, preferisce piuttosto dibattere il tema grammaticale, proponendo e suggerendo, nel convincimento che la lingua non sia governabile dall'alto e che il potere regolativo di un linguista, sia pure autorevole, si riduca fatalmente ai settori meno vincolati al ceppo della tradizione (nomenclature settoriali, formazione delle parole, ecc.). Se egli

4. Migliorini ricorda qui una voce di sapore goldoniano (morbino) e una d'area meridionale (pittare), segnalando la scarsa fortuna toccata in genere ai dialettalismi che esprimono «sfumature affettive, caratteri, azioni, sentimenti intrisi di color locale», rispetto a quelli che rimandano a cognizioni oggettive. Cf. anche Migliorini (1978: 410).

5. Si pensi a Ugolini (1858), a Fanfani e Arlia (1881) e a Rigutini (1905).

6. Sulla sensibilità di Migliorini alle ricerche lessicali insiste da ultimo Serianni (2009: 10-11). 
accoglie senza limitazioni un neologismo lessicale, lo fa perché ritiene che esso sia necessario, e comunque non alteri l'organismo linguistico, né urti la sensibilità grammaticale dei lettori. Gli interessa la funzionalità comunicativa dell'italiano, una lingua che ancora nel primo Novecento aveva sostanzialmente un unico codice, quello letterario stabile nella sua struttura.

Anche quando censura voci dialettali, stranierismi inutili, locuzioni malformate e coniazioni poco felici, si astiene dall'invocare interventi normativi sulla lingua; con l'assumere come criterio discriminante quello strutturale e funzionale, egli finisce piuttosto con l'allargare il canone di quei grammatici e linguisti che, ancora in pieno Novecento, si mostravano riluttanti a ogni sollecitazione centrifuga rispetto alla lingua letteraria ${ }^{7}$. A questa Migliorini non esita a riconoscere la preminenza: «la lingua letteraria del tempo nostro ha diritto al primato, ma non alla dittatura» (editoriale «Propositi», in Lingua Nostra II, 1940: 1), ma egli sa bene che gli altri materiali linguistici, siano essi riconducibili al dialetto o alle lingue speciali, hanno una loro giustificazione e non vanno giudicati meno legittimi della lingua letteraria: materiali distanti sì dalla lingua comune, ma non per questo vitandi.

\section{Tendenze neopuristiche nell'analisi di stranierismi e neologismi}

\section{Ma vediamo più davvicino alcuni interventi di Migliorini.}

Se è vero che il linguista rodigino può definirsi un moderato neopurista ${ }^{8}$, rispettoso delle proposizioni dei lettori di un giornale, appartengano essi a strati più o meno colti, è anche vero che significative appaiono le sue argomentate deflessioni dai precetti del rigorismo puristico ${ }^{9}$. Diversamente dai puristi, vecchi e nuovi, egli non è pregiudizialmente ostile a ogni tipo d'innovazione, ma allarga l'orizzonte d'interesse oltre i fatti lessicali, ammettendo i prelievi dalle lingue speciali e intuendo la caducità di molti dei neologismi inutili o male foggiati, destinati a uscir d'uso, indipendentemente dalle proibizioni dei grammatici.

Talora, la dissociazione tra lo statuto linguistico e l'uso effettivo emerge chiaramente: a Odoardo Gori, sostenitore di autarcia come unica forma corretta (Settegiorni, 1 agosto 1942), Migliorini riconosce sì la validità della norma di pronunziare le parole greche secondo le regole latine, ma obietta

7. Basterà ricordare il manuale di Gabrielli (1956) che conobbe un largo successo di pubblico. Uguale fortuna arrise ad altre sue opere divulgative (cf. Gabrielli, 1976, 1977, 1978).

8. Il termine Neopurismo fu adoperato per la prima volta proprio da Migliorini nell'Archivio glottologico italiano XXVII (1935). Per la definizione di Neopurismo, prendiamo a prestito le parole dello stesso Migliorini («Tendenza ad escludere dalla lingua quelle voci straniere e quei neologismi che siano in contrasto con la struttura della lingua, favorendo, invece, i neologismi necessari e ben foggiati: si tratta di un tentativo di applicazione degli insegnamenti della linguistica a un moderato purismo») ricordate da Castellani (1979: 26-27). In quest'accezione, a parere di Castellani, sarebbe meglio parlare di purismo strutturale (Castellani, 1979: 28). Si vedano le osservazioni di Ghinassi (1990: LIX-LXX) e la bibliografia citata alla nota 33; il capitolo Purismo e neopurismo in Migliorini (1990: 81-107). Le vicende del neopurismo miglioriniano sono ricostruite ora con dovizia di particolari da Fanfani (2009: 56-67).

9. Castellani distingue il purismo bembiano e il purismo tradizionale che si oppone alle tendenze innovative, «attenuandosi sempre più il primo a favore del secondo» (Castellani, 1979: 28). 
che autarchia è legittimata dal consenso generale e vano risulterebbe ogni tentativo di sbarrarle il passo. Una sentenza latina conforta e suggella il suo discorso: error communis facit ius ${ }^{10}$.

Ai presupposti teorici del purismo sembra rinviare l'atteggiamento di Migliorini quando disapprova nella lingua dei giornali la diffusione di succube a scapito di succubo ${ }^{11}$, unica forma corretta da connettere con incubo; «la forma erronea è dovuta al fatto che non si è ricorsi direttamente al latino, ma al francese succube» (Tempo, 21 luglio 1948). È questo uno dei casi in cui Migliorini difende puristicamente la variante minoritaria, rifiutandosi di piegarsi all'uso prevalente. La sua posizione ricompare alcuni anni dopo nelle pagine di Gabrielli (1977: 79-80). Meno tradizionalisti appaiono, invece, i linguisti dei giorni nostri: se Della Valle e Patota (2000: 62-63) ammettono senza riserve l'oscillazione morfologica, Luca Serianni la confina nella zona grigia della norma linguistica, avvertendo che succubo «è la forma più tradizionale e raccomandabile» (Serianni, 1989: 196) ${ }^{12}$.

Entro il recinto del purismo classico può rientrare l'atteggiamento di Migliorini quando lamenta la fresca coniazione di settennato («comincia a far capolino»: Tempo, 20 maggio 1948). A suo parere, per la nuova esigenza terminologica, l'indicazione del periodo fissato dalla Costituzione per la durata della carica del Presidente della Repubblica, settennio, voce dotta che ben si adatta alla struttura dell'italiano, è preferibile a settennato, che ha il difetto d'essere ricalcato sul francese septennat. Né si avverte la necessità, argomenta lo studioso, di distinguere con due vocaboli diversi il «periodo di sette anni» (settennio) e «sette anni di carica» (settennato).

Il tentativo di Migliorini di bloccare sul nascere lo stranierismo, com'è noto, non è stato coronato da successo; oggi il francesismo settennato ha avuto la meglio, relegando settennio a variante rara e, per questa ragione, di tono un po' letterario. Mette conto ricordare che il sentimento linguistico di Migliorini, così saldamente ancorato alla tradizione, affonda le radici nella sensibilità nazionalistica che è presente in tanta cultura di primo Novecento e che negli anni si è progressivamente attenuata; ma immutata rimane la validità del suggerimento che per i bisogni terminologici l'italiano si rifaccia in prima istanza alla lingua madre, il latino.

La recensione della seconda edizione di Monelli (1943) offre a Migliorini l'opportunità di segnare qualche distanza dai tentativi di epurazione che punteggiano le pagine di quel fortunatissimo repertorio. Questo non significa che si dissolva d'un tratto la diffidenza per i forestierismi di più recente e più larga diffusione, specie per quelli difformi dalle strutture linguistiche tradizionali; egli non se la sente «di salvare dall'ostracismo, come per una volta tanto il Monelli vorrebbe, la parola brioche»

10. Per la storia della parola autarchia cf. Migliorini (1990: 227-236). Oltre al Gori, anche Vincenzo Costanzi e Vittorio Brondi preferivano autarcia (Migliorini, 1990: 235, nota 27).

11. «Se contiamo le volte in cui si legge nei giornali succube, ci accadrà di aspettare otto o nove volte prima di imbatterci nella forma corretta succubo» (Tempo, 21 luglio 1948).

12. Cf. anche Serianni (2004: 90); il foglio La Crusca per voi nº 23 (2001), p. 7. 
(Settegiorni, 27 febbraio 1943), che va emendata a causa di un gruppo consonantico che mal si accorda col sistema fonologico italiano ${ }^{13}$.

Al suo sentimento linguistico corrispondono meglio quegli adattamenti che non presentano difficoltà fonologiche: Migliorini non obietta nulla contro gilè e bistecca, parole concrete e d'uso popolare, ma in linea con Monelli biasima overtura, parola colta e di carattere astratto, sentita come surrogazione di ouverture, poco felice sotto il rispetto morfologico e mal rispondente alle necessità dell'epoca. I fatti gli hanno dato ragione; «proposto nel 1939 da una commissione autorevole» (Migliorini, 1990: 105), il termine overtura non ha mai conosciuto l'approvazione dell'uso, a differenza di gilè e bistecca che hanno stabilmente attecchito. Né andrà tralasciata l'aura di raffinata eleganza propria di una voce come ouverture, a fronte di un adattamento che sin dalla nascita aveva l'aria di non tener conto delle necessità strutturali dell'italiano: merita ricordare inoltre come i Francesi nel recente passato abbiano goduto di molta considerazione come arbitri di eleganza. Gl'intellettuali di primo Novecento, se poterono tollerare l'esoticità di ouverture, non esitarono a cassare overtura come idiotismo spurio.

Rispetto ai forestierismi, più ampi confini sono concessi da Migliorini, e in genere dai neopuristi, ai neologismi, specie a quelli derivativi, che cominciavano a far capolino nel linguaggio giornalistico, desideroso spesso di arricchire le nomenclature esistenti. A proposito di kartismo (foggiato sopra kart e go-kart, secondo il modello di automobilismo e ciclismo), che prevedibilmente urtava la coscienza linguistica di molti Italiani dell'epoca, lo studioso interviene con brevi osservazioni, considerandolo rispettoso della struttura dell'italiano e poco esposto al rischio di confusione con gli omonimi (Corriere della Sera, 11 ottobre 1960). Nel giudicarlo neologismo accettabile, Migliorini osservava criteri esclusivamente linguistici (la necessità significativa del termine, la sua conformità alle strutture morfologiche, il principio dell'analogia), lasciando da parte i fattori culturali e stilistici (l'ostilità, di matrice nazionalistica, verso il $K$, estraneo all'alfabeto italiano e la coesistenza di parole uguali nel significante) che tanta parte avevano avuto nelle proibizioni puristiche. Nell'italiano contemporaneo automobilismo e ciclismo si sono ormai stabilmente impiantati: lo stesso non può dirsi per Kartismo e altre formazioni derivative (Kartista e Kartodromo), linguisticamente motivate, ma da molti sentite come poco rispettose del carattere originario (verrebbe da dire: del «genio») della nostra lingua ${ }^{14}$.

Consapevole che il significato di una neoformazione può esser chiarito solo dall'uso, Migliorini apre volentieri al rinnovamento lessicale della nostra lingua; così, ad esempio, intuisce felicemente che carrello o carrellino per la spesa (Corriere della Sera, 18 gennaio 1961) è destinato a imporsi sul concorrente passeggino alimentare e anche su portaspesa, che pure ha il pregio d'essere una parola sola.

13. Sull'adattamento degli stranierismi cf. anche le puntuali osservazioni di Migliorini (1990: 104).

14. Sorte diversa è toccata a voci come autodromo e velodromo penetrate in italiano dal francese. 
Posizione accomodante Migliorini esprime nei confronti di estrogeno (Corriere della Sera, 2 agosto 1960), che all'epoca era già un neologismo integrato. La parola doveva sembrargli poco trasparente soprattutto nella prima delle sue due componenti (estro-); la seconda (-geno «che genera») aveva da tempo una certa diffusione e notorietà. Migliorini osserva come estro- non vada confuso con l'elemento compositivo di parole come estromettere ed estroflesso, ma continui il greco oistros («tafano», e metaforicamente «veemente pazzia»). Situazione analoga in latino: oestrus aveva il significato di «tafano» e di «furore poetico», negli scrittori cristiani quello di «foga amorosa». Questo significato, conclude Migliorini, doveva essere ben presente sia al Petrarca che parla di amorose vespe per indicare la passione d'amore ${ }^{15}$, sia al naturalista che nel Novecento conia estrogeno.

Perché Migliorini ritiene che si possa accogliere questo tecnicismo, prima riferito alle sostanze che provocano l'estro venereo e poi reso popolare dall'industria avicola? Perché egli sa bene che la scienza e la tecnica da sempre sono esposte all'ibridazione linguistica e che l'italiano e ancora prima il latino per le necessità terminologiche hanno attinto al serbatoio del greco, lingua concettualmente ricca e più di altre aperta alla composizione lessicale ${ }^{16}$. Non c'è dunque fastidio per l'innovazione lessicale, c'è piuttosto attenzione a un concetto moderno che trova compiuta espressione in una neoformazione di conio classico. Come altre parole della scienza, estrogeno scaturisce da un processo formativo che esalta le capacità onomaturgiche di chi rivitalizza elementi lessicali di un'età remota per esprimere una nuova nozione. L'italiano, lingua di solida cultura umanistica, ha incorporato facilmente estrogeno e lo ha utilizzato volentieri anche per il ritmo dattilico proprio delle parole proparossitone ${ }^{17}$.

A un settore tradizionalmente esposto alla moda appartiene glamour, un esotico angloamericanismo che mantiene a tutt'oggi una certa popolarità. Migliorini ne sconsiglia l'uso: glamour («incantesimo») e grimoire («libro di stregoneria») rappresentano la forma che la parola grammatica ha preso rispettivamente in Scozia e in Francia e per questo hanno sapore di localismi. In Francia, all'àmbito delle arti magiche - osserva lo studioso - rimandava originariamente anche charme, che a partire dal Seicento sarà riferito alla magia della bellezza femminile: allargando il significato, charme entrerà poi a far parte delle parole comuni. Analogamente, in Italia hanno assunto via via significato più ampio voci come incanto, incantesimo, fascino e malia, che negli scrittori più antichi erano regolarmente accostate a operazioni di magia. Passando dall'idea ristretta di «formula magica» a quella di «bellezza incantatrice» e a quella più generica di «fascino», glamour, a dire di Migliorini, avrebbe compiuto un percorso simile a quello di fascino e malia: di qui il suggerimento che rispetto allo stranierismo siano preferibili i corrispondenti italiani che lo traducono «abbastanza bene» (Corriere della Sera, 23 dicembre 1960) $)^{18}$.

15. Petrarca, Canzoniere, CCXXVII, v. 5-6 («tu stai nelli occhi ond'amorose vespe/ mi pungon sì, che 'nfin qua il sento et ploro»). Su questa e altre metafore petrarchesche cf. Migliorini (1978: 207).

16. L'argomento è largamente dibattuto in Nencioni (1997: 1-4).

17. Sulla facilità dell'italiano nell'utilizzazione di parole sdrucciole cf. Migliorini (1973: 260).

18. Analoga posizione ritroviamo alcuni anni prima nella grammatica di un illustre accademico d'Italia, Alfredo Panzini, 
L'intervento di Migliorini segnala che alla metà del secolo scorso glamour era estraneo al sentimento linguistico degl'Italiani e che la sua presenza non rispondeva ad alcuna necessità terminologica, trattandosi peraltro di voce inadatta ad assumere un preciso compito tecnico che potesse giustificarne l'uso. Si tratti di lingue neolatine o piuttosto lingue di struttura e tradizione diverse, di francese o inglese, il suo atteggiamento nei confronti della parola avventizia è sempre ispirato a cautela.

Cautela eccessiva? Non credo, così come non credo che nei parlanti e negli scriventi di ieri e di oggi l'uso di glamour abbia mai conosciuto esiti denotativamente produttivi: quel che è rimasto intatto è semmai l'alone connotativo da taluni ravvisato nel termine. La nota di Migliorini richiama alla nostra memoria un'importante nozione: il mutamento di significato rappresenta un fenomeno che è proprio del funzionamento dell'organismo linguistico, e come tale va accettato ${ }^{19}$; nel proporre parole cariche di anni come fascino e malia, egli vuole solo difendere con orgoglio la lingua nazionale e la cultura che in essa si esprime. Nulla di più, nulla di meno: le angosce proibizionistiche tipiche dei puristi appartengono non più che tanto al suo sentire ${ }^{20}$.

Non è raro nelle rubriche linguistiche dei giornali dell'epoca un certo desiderio di normativismo razionalizzante, ispirato dall'idea che la norma della lingua debba poggiare esclusivamente sulla logica; atteggiamento, questo, ben presente anche nei giornali di oggi. Accade così che un lettore (Corriere della Sera, 13 settembre 1960) disapprovi semianalfabeta, come un prefissato che per graduare l'intensità semantica della parola base finisce con l'attenuarne in modo incoerente il connotato negativo, e gli preferisca parzialmente alfabeta e semialfabeta. Conscio che la lingua abbia confini più ampi della semantica, Migliorini storicizza il problema, adducendo qualche utile testimonianza: analfabeta si è imposto saldamente nell'uso popolare come epiteto ingiurioso ed è passato dall'italiano (non direttamente dal greco) in francese; alfabeta è apparso più tardi in opposizione ad analfabeta, ma non è riuscito a penetrare nell'uso quotidiano; c'è in semianalfabeta «un implicito disprezzo» sconosciuto alle altre formazioni, che pure rispondono coerentemente alla semantica.

Quali insegnamenti si ricavano dalle osservazioni di Migliorini? Almeno due: la norma linguistica si fonda sull'uso dei parlanti e degli scriventi; la spontaneità della lingua va tenuta al riparo da occhiuti scrupoli razionalistici che sanno un po' di pedantesco ${ }^{21}$. Semianalfabeta è forse superfluamente contraddittorio ma espressivo, preferibile a parzialmente alfabeta e semialfabeta, voci distanti dal comune sentimento linguistico, ascrivibili a un atteggiamento logicizzante impropriamente applicato al vocabolario.

che scorge «una certa goffàggine da provinciali in questo non sapersi esprìmere senza domandare la carità delle parole alle altre nazioni» (Panzini, 1933: 84). Di glamour si parla anche in Lingua Nostra XXIX (1968), p. 32.

19. Si veda in proposito quel che dice Giovanni Nencioni: «ll cambiamento di significato appartiene alla fisiologia delle lingue e quindi, come fenomeno linguistico, non può essere respinto» (La Crusca risponde, 1995: 47).

20. «Còmpito dei cultori della lingua è non quello puramente negativo di combattere contro il forestierismo, ma di cercare o addirittura di coniare il termine che occorre» (Migliorini, 1990: 91-92).

21. Si vedano, in proposito, le osservazioni di Serianni (2004: 95-97). 
Restiamo nell'àmbito della semantica. Nel Tempo del 19 settembre 1948 si segnala l'uso sinonimico che i giornali sportivi fanno di olimpico e olimpionico (gare olimpiche e gare olimpioniche). L'errore sollecita la riflessione di Migliorini sull'origine delle due parole: olimpico («che riguarda Olimpia») è un aggettivo generico, che copre uno spazio semantico più ampio rispetto a olimpionico («vittorioso in Olimpia»), voce composta, usata anticamente come sostantivo più che come aggettivo. Stando all'etimologia dunque, le due parole non possono avere lo stesso significato ${ }^{22}$. A proposito di olimpico, lo studioso segnala inoltre un equivoco, non raro presso parlanti e scriventi anche dei nostri giorni: il riferimento che nelle espressioni Giove olimpico, calma olimpica alcuni fanno all'Olimpo piuttosto che alla serenità espressa dalla famosa statua criselefantina di Giove, opera di Fidia conservata nel tempio di Olimpia ${ }^{23}$. Di olimpionico non esita infine ad accettare la pronuncia sdrucciola, pure avvertendo che l'accento originario della corrispondente voce greca richiederebbe la pronuncia piana.

Nei tempi moderni nessuno pensa di esumare la pronuncia etimologica piana per olimpionico e tantomeno per una parola come America; se olimpionico ha facilmente attecchito inserendosi nel nutrito drappello di aggettivi sdruccioli in -ico (antropico, ematico, ippico, ecc.: cf. Migliorini, 1990: 199-212), si deve al fatto che la lingua è un sistema, talora incoerente, nel quale possono coesistere vari registri: il grammatico Migliorini si è in questo caso limitato a prendere atto della concreta situazione, consapevole che le ragioni dell'uso sono destinate prima o poi a prevalere su quelle astratte dell'etimologia.

Una poco felice formulazione nel regio decreto del 2 ottobre 1930 ( «la denominazione del Comune di Spezia è rettificata in La Spezia») offre a Migliorini l'opportunità di segnalare la presenza dell'articolo nell'esatta scrizione dei nomi di due città: la Spezia e l'Aquila (Settegiorni, 31 ottobre 1942). Il frequente uso erroneo delle preposizioni articolate e la presenza della maiuscola nell'articolo (di La Spezia, de La Spezia, ne La Spezia, ecc.) gli appaiono direttamente riconducibili a coloro che hanno interpretato alla lettera il testo di quel decreto; meglio hanno fatto quanti, assecondandone piuttosto lo spirito, hanno spontaneamente detto e scritto nella Spezia, della Spezia.

Il discorso non può dirsi chiuso, se ancora qualche anno fa il foglio La Crusca per voi ospitava la lettera di Paolo Emilio Faggioni, un professore spezzino intento a difendere l'articolo nel nome della sua città e a segnalare la circolazione di «espressioni arbitrarie ed aberranti quali di La Spezia, a La Spezia, ecc.»²4 (La Crusca risponde, 1995: 198). La risposta di Piero Fiorelli, consulente di turno dell'Accademia della Crusca, alle lagnanze del citato professore è ricca di motivazioni grammaticali, oltre che giuridiche; in linea con Migliorini, anche Fiorelli si preoccupa di storicizzare la questione,

22. Cf. anche Lingua Nostra I (1939) p. 109 e Migliorini (1990: 196). L'uso dei termini continua a suscitare dubbi e incertezze se prima Gabrielli (1977: 77) e poi Della Valle e Patota (2000: 74-75) tornano sull'argomento.

23. Abbondanti attestazioni dell'uso figurato di olimpico sono leggibili nel GDLI (s.v., nº 8: Giusti, De Sanctis, Ghislanzoni, Bersezio, Carducci, Nencioni, Tarchetti, Bechi, Cicognani, Slataper, Baldini).

24. Sulla presenza dell'articolo determinativo nei nomi di città si vedano le osservazioni di Gabrielli (1976: 143-44). 
fissando l'obiettivo proprio sul regio decreto e in particolare sull'ambiguità dell'articolo maiuscolo (La Spezia): di qui sarebbe discesa, in «molti lettori sprovveduti, in primo luogo burocrati e statistici» (La Crusca risponde, 1995: 199) l'idea che la città avesse un primo (La) e un secondo nome (Spezia).

Quale morale ricaviamo da una questione così a lungo dibattuta? L'attualità delle parole di Migliorini rivive nell'intervento di Fiorelli: una maggiore attenzione alle ragioni dell'uso linguistico della collettività forse avrebbe sconsigliato all'estensore del decreto la perentorietà dei toni. Se ci si fosse limitati a prescrivere il nome della città con la minuscola dell'articolo (la Spezia), così come suggeriva Migliorini in Settegiorni, con ogni probabilità le proporzioni dell'uso erroneo sarebbero state inferiori. Di là dal caso specifico, nei suggerimenti di Migliorini appare già ben viva l'esigenza di stile semplice nelle comunicazioni del potere, emersa poi prepotentemente sul declinare del secolo scorso e attuale anche ai giorni nostri ${ }^{25}$.

Di lingua burocratica e grammatica si parla nella Nazione dell'11 agosto 1954. L'intento del Ministero delle poste e telecomunicazioni di regolamentare il computo delle parole nei telegrammi e un elenco di circa 1200 voci trasmesso agli uffici postali sono per Migliorini l'occasione per parlare dello stile telegrafico e della sua ricaduta sulla lingua di tutti i giorni. La sua attenzione va alle notizie giornalistiche spesso pubblicate nella forma originaria e priva di nessi della trasmissione telegrafica; si deve a questa consuetudine, se parole-macedonia come Bankitalia e Comit «tendono disgraziatamente a penetrare nell'uso corrente». La reattività di Migliorini è quella tipica dello studioso istituzionalmente chiamato alla difesa della buona lingua; andrà notato tuttavia che egli si è rivelato davvero un buon profeta: pure di diversa trasparenza, quei compendi telegrafici ricorrono a ogni passo negli odierni mezzi di comunicazione di massa (rubriche radiofoniche e televisive, inserti giornalistici, ecc.) e sono ritenuti facilmente intelligibili26.

Insieme con le espressioni abbreviate, anche le parole composte davano corpo all'elenco del Ministero. Migliorini le passa in rassegna minutamente con la consueta acribia; di qui scaturisce il compiacimento per l'assenza dell' «orrendo contrassegno»; la perplessità per la presenza di raccattapalle, sopreccellente e tirinnanzi; la sollecitazione per l'accoglimento avvenire di voci come giallorosso, grigioverde, sordomuto e canelupo. Fondamentalmente, lo studioso sembra condividere l'aspirazione a una qualche certezza normativa, presente nell'iniziativa del Ministero; egli sa bene tuttavia che il tentativo di fissare regole certe, anche là dove vige l'incertezza, racchiude fatalmente una certa quota di arbitrio: non può far altro quindi se non prendere atto che per il compilatore ministeriale nullaosta e collurgente contano per una parola, casellapostale e vagonletto per due.

Colpisce la sensibilità linguistica di Migliorini il corto circuito geometrico di convergenze parallele, espressione enigmatica attribuita ad Aldo Moro, frequente nel linguaggio politico degli anni Sessanta e Settanta del Novecento. Ancor di più lo urta l'utilizzazione incoerente di metafore come il

25. Basti pensare a Cassese (1994), a Franceschini e Gigli (2003) e a Fioritto (2009).

26. Della tendenza all'economia intrinseca alla lingua si parla più diffusamente in Migliorini (1990: 10, 42-43). 
rimpasto è prossimo al varo; a un certo specchietto è difficile abboccare; i leoni corrono col contagocce; nell'audace e inusuale accostamento di parole prive di alcun rapporto di somiglianza egli scorge i segni di una cattiva coscienza linguistica, sorda ai precetti dell'antica retorica che «è stata messa nel dimenticatoio» (Corriere della Sera, 2 agosto 1960). Verosimilmente, le metafore costruite, proprie dello stile brillante di qualche giornalista dell'epoca, appaiono a Migliorini un ostacolo per tutti coloro che aspirano a esprimere e comunicare compiutamente il proprio pensiero. Se alla base del rifiuto di ogni affettazione c'è il gusto per una lingua più vera e spontanea, questo non modifica la distanza che lo separa dal purismo più intransigente, specie nella dimensione preconcetta dell'avversione per ogni uso metaforico.

Qui si può ricordare la sua nota su cipolla (o cipollone) «vecchio orologio di poco valore» (Corriere della Sera, 13 settembre 1960). Migliorini non condivide l'idea dei lessicografi che spiegano l'origine della metafora attraverso il riferimento alla presunta grossezza dell'orologio, ma, com'è suo costume, procede in punta di piedi e riduce al minimo la portata del suo dissenso. Egli esamina in prospettiva storica il significato accessorio assunto dall'italiano cipolla e dal corrispondente francese oignon e va a cercarlo nel periodo e nell'ambiente in cui esso comincia a farsi strada: le parole vengono ricondotte nell'àmbito del Settecento, quando era in uso un orologio che veniva custodito in una cassetta di tre strati: «prima di poterlo guardare bisognava togliere l'uno dopo l'altro due involucri». Naturale che il pensiero di molti, conclude Migliorini, andasse al bulbo della cipolla²7.

La storia di cipolla nel suo significato secondario ci conferma che anche nel lessico non intellettuale si può scorgere qualche riflesso della storia culturale di un popolo; in questo caso, la congruenza semantica di cipolla e oignon non è certo una coincidenza casuale, ma è facilmente attribuibile alla tradizionale solidarietà tra due aree aperte a lingue sorelle: per spiegare i vari significati assunti da una parola, l'abito della storicizzazione si rivela ancora una volta come il più adatto. Se all'altezza cronologica della noterella di Migliorini cipolla «orologio» era accezione non rara nella lingua quotidiana, almeno negli usi marcati, oggi la metafora sopravvive nel registro scherzoso di esigue fasce di parlanti.

Anche in materia di meccanismi derivativi, Migliorini appoggia le sue tesi su basi storicamente fondate. Un esempio: un lettore del Corriere della Sera (21 ottobre 1960), incline a credere che le astratte ragioni del processo derivativo possano prevalere sulla realtà dell'uso, eccepisce sulla connotazione negativa comunemente attribuita a reazionario, dal momento che la parola deriva da una base qualificata non negativamente come reagire. Migliorini prende spunto dall'eccessivo logicismo del lettore per segnalare come nella concreta esperienza della lingua siano rari i derivati che mantengono il significato della parola base; e a sostegno del suo assunto, egli cita altri derivati di reagire (reattore, reagente, ecc.) ricadenti in àmbiti disparati. Storia tutta diversa quella di reazionario che nasce in Francia all'epoca della Rivoluzione, «con significato esclusivamente politico» e in accezione pole-

27. Altre osservazioni si possono leggere in Lingua Nostra XV (1954), p. 89. 
mica: significato e accezione che la parola, pur con qualche incertezza, ha conservato fino ai nostri giorni.

In Settegiorni del 22 agosto 1942 si parla dell'abitudine tutta italiana di coniare parole erudite e altisonanti per esprimere cose quotidiane ${ }^{28}$. Lo spunto è fornito dall'iniziativa dell'Opera Maternità e Infanzia rivolta alla preparazione di giovani donne abili nell'allevamento di bambini: per definirle Migliorini trova inadatti sia i tradizionali nomi di bambinaia e balia asciutta, sia gli esotici bonne e nurse; né lo convincono le proposte avanzate nelle pagine dei giornali dell'epoca: nutrice, vigilatrice, vicemadre, governante, allevatrice. La sua preferenza va ad allevante, allevabimbi e soprattutto all'aggettivo sostantivato materna, che «ricorderebbe, senza usurparlo, il dolce nome di madre, e ricorderebbe l’Opera Maternità e Infanzia che a questa vigilanza presiede».

Quelle di Migliorini sono le proposte concrete del linguista che, ossequente ai criteri del neopurismo, considera la funzionalità dei neologismi per rispondere alle necessità onomasiologiche della società del tempo; allevante, allevabimbi e materna sono tutte voci carezzevoli conformi alle norme strutturali dell'italiano, ma questo non è bastato perché esse attecchissero: il consenso nel loro uso non c'è mai stato. Rappresentate ora da una neoformazione (allevabimbi), ora da una nuova utilizzazione di parole già esistenti nell'italiano (allevante, materna), le soluzioni del problema glottotecnico dovettero rivelarsi, forse, poco espressive e comunque inidonee a soddisfare i bisogni della cultura coeva. Non solo: se la resistenza del sentimento linguistico di Migliorini è stata efficace nel contrastare la coniazione di una voce pedantesca, poco ha potuto contro l'accoglimento di uno stranierismo come baby-sitter, che nel secondo Novecento avrebbe soppiantato bambinaia, coprendo un àmbito sociologico e professionale diverso e fornendo un modello per analoghe formazioni (dog-sitter, cat-sitter, ecc.), talvolta capricciose ed effimere (nonna-sitter, nonno-sitter, ecc. ${ }^{29}$.

Nella Nazione del 17 febbraio 1955 Migliorini si chiede se in Italia accanto al nome di (ferrovia) metropolitana possa circolare nella lingua di tutti i giorni un nome accorciato, così come è accaduto in Francia, dove chemin de fer métropolitain si è ridotto prima a le métropolitain e poi a le métro. I possibili concorrenti di metropolitana non lo convincono: «pessima» soluzione giudica il metrò, per l'accento e il genere maschile propri del francese; ancora meno lo soddisfa il metro, voce diffusa in italiano ma in un altro significato; un po' più intonata gli appare la brevità di la metro, che verrebbe a far parte di una serie fortunata: la foto (da la fotografia), la moto (da la motocicletta), ecc. In mancanza di un sinonimo semplice ed espressivo, Migliorini si volge ancora una volta indietro e pensa a una parola già esistente nella nostra lingua. Nel proporre la sotterranea, egli suggerisce di affiancare alla

28. Ancora negli anni Sessanta del Novecento, Emilio Peruzzi tornava sull'argomento lamentando l'assenza di «un vocabolario comunemente accettato ed univoco per parlare delle mille piccole cose della vita di tutti i giorni» (Peruzzi, 1961: 15).

29. Per Durante baby sitter rientra tra gli anglismi non adattati «accessibili all'uomo della strada» (Durante, 1982: 266). Sempre attuali le parole con cui Migliorini chiudeva il saggio Anteguerra-Dopoguerra: «ll neologismo capriccioso ricade spesso nell'ombra, quello che dà forma a un nuovo concetto largamente diffuso entra saldamente nel lessico» (Migliorini, 1990: 226). 
parola «ufficiale» (la metropolitana) una parola dello stesso tipo, un aggettivo sostantivato, sentito però come un termine popolare, rispondente alle sopravvenute esigenze della popolazione della grande città e per questo utile sotto il rispetto sociale, oltre che linguistico.

Successiva all'inaugurazione della ferrovia metropolitana di Roma, la nota miglioriniana su metropolitana è ispirata dalla consapevolezza che avvenimenti siffatti rappresentano il momento decisivo per il mutamento linguistico. L'analisi che egli fa degli improbabili candidati alla successione di metropolitana non è solo la consueta e puntuale descrizione della lingua in movimento, è anche e soprattutto la testimonianza di un robusto senso della lingua come istituzione sociale.

Mi piace ricordare infine una minuzia grammaticale, una noterella in materia di ortografia, che, com'è ben noto, da taluni è ancora oggi considerata un capitolo secondario della grammatica: basti pensare alla poca attenzione dedicata da qualche grammatica scolastica all'uso corretto di segni grafici e interpunzione. Nel Tempo del 10 novembre 1948 Migliorini segnala «un antipatico errore»: li accentato nelle date (li 15 settembre). L'origine dell'errore, osserva Migliorini, è chiarita da coloro i quali, mettendo la virgola dopo li (li, 15 settembre), finiscono con lo sbagliare in modo ancora più evidente: chi scrive così mostra di confondere con l'avverbio di luogo li l'antico articolo determinativo plurale $l i$, ancora presente in parecchi dialetti e rimasto a lungo nella lingua letteraria, specie dopo la preposizione per $^{30}$. Questa la conclusione di Migliorini: Ii 15 settembre equivale a i 15 settembre e non c'è ragione alcuna per accentare qui e altrove l'articolo.

Un lungo lasso di tempo ci separa ormai dalla nota miglioriniana e l'insegnamento della scrittura, almeno nei corsi universitari, è oggi meno empirico rispetto al passato; li con l'accento nelle date tuttavia resiste e appare presso categorie di scriventi che pure sono ben capaci di controllare l'ortografia, il ramo della grammatica in cui la presenza di una norma più sicura lascia tradizionalmente pochi spazi a incertezze e ambiguità. Il costrutto sente ormai di stereotipo e si è diffuso perché molti, suggestionati dalle scritture burocratiche dove li appare sovente nelle date, hanno, per dir così, automatizzato la scrittura scorretta, allontanandosi dalla scrittura con l'articolo plurale, ormai desueta e di tono decisamente letterario ${ }^{31}$. La lingua delle lettere circolari ha contribuito, forse, a tenere in vita l'errata immagine grafica del monosillabo in un settore, quello della scrittura, capace di alimentare da sempre la memoria visiva delle parole. Quanto sia tenace questo tipo di memoria ce lo dicono i tanti scriventi che nelle lettere ancora oggi ricorrono erroneamente a li accentato col verosimile intento di segnalare il luogo da cui scrivono.

30. Una sopravvivenza di quest'uso antico si scorge nelle forme per lo meno e per lo più.

31. Della Valle e Patota definiscono l'articolo li «un'anticaglia» (Della Valle e Patota, 2000: 123). Per Gabrielli è «muffitissimo» (Gabrielli, 1976: 148). 


\section{L’azione educatrice di Migliorini}

È tempo di raccogliere le fila del discorso. La nostra ricognizione testimonia l'attenzione di Migliorini giornalista al naturale processo evolutivo della lingua. In lui la capacità di leggere il presente e di spiegare le tendenze in atto si salda con quella dello studioso dell'intera tradizione della nostra lingua, sapientemente messa a frutto nella produzione maggiore: ciò che più colpisce è come anche la nota lessicale più breve, presentata con l'abituale discrezione, poggi su una solida base di erudizione e su una fitta rete di riscontri con le altre lingue, anche con quelle più appartate. Rispetto a puristi e classicisti, Migliorini ha un orizzonte più ampio, perché guarda alla lingua tutta, non solo a quella letteraria; il suo gusto democratico emerge dal tentativo d'individuare il legame tra lingua della comunicazione cólta e lingua pratica, tentativo non facile che risponde all'intento di soddisfare le esigenze terminologiche di quanti fanno fatica ad esprimere compiutamente le inesorabili realtà quotidiane. Sono, come si è visto, interventi anche minimi ${ }^{32}$ che rivelano tuttavia, non diversamente dai grandi saggi, la straordinaria capacità del divulgatore, già autorevolmente messa in luce: a partire da Giovanni Nencioni che ha parlato di «vocazione didattica» (Nencioni, 1976: 28), fino a Manlio Cortelazzo che ha insistito sulla «missione educativa e pedagogica del grande pubblico» ${ }^{33}$ (Cortelazzo, 2009: 251).

Azione educatrice dunque quella di Migliorini, sollecitata dal desiderio che i lettori dei giornali dell'epoca, non sempre capaci di usare l'italiano con sufficiente padronanza, prendano finalmente coscienza del valore della loro lingua. Ancora prima che la televisione favorisse l'estensione dell'uso dell'italiano, lo studioso rodigino ha provato a insegnare agl'Italiani il funzionamento della lingua: attraverso le sue specole linguistiche egli ha dialogato e comunicato coi suoi lettori in rapporto autentico e schietto, rivelandosi consulente ligio nell'osservanza delle norme, pronto a cimentarsi fuori dai recinti accademici nel terreno insidioso dell'azione sociale. Distinguere l'uso antiquato dal corrente, leggere grammatica e vocabolario con la mente sgombra da pregiudizi logicistici e insieme credere nella forza assimilatrice dell'italiano sui forestierismi è stato il cardine delle sue conversazioni giornalistiche.

32. A proposito degli articoli da Migliorini pubblicati nei giornali e poi raccolti nel volume Profili di parole, Luca Serianni ha scritto: «ll tono amabilmente discorsivo non inganni: si tratta di brevi o brevissimi articoli fondati su ricerche vaste, di prima mano, guidate da un senso linguistico sempre vigile e acuto» (Serianni, 2009: 12).

33. Si segnalano anche le osservazioni di Ghinassi (1979: 47), Viale (2009: 291 ss.), Serianni (2012: 54-5). 


\section{Bibliografia}

Baldelli, Ignazio (1979), «Bruno Migliorini studioso della lingua italiana», in Massimo Luca Fanfani (ed.), L'opera di Bruno Migliorini nel ricordo degli allievi, con una bibliografia dei suoi scritti, Firenze, Accademia della Crusca, p. 17-22.

Cassese, Sabino (ed.) (1994), Codice di stile delle comunicazioni scritte ad uso delle amministrazioni pubbliche, Roma, Istituto Poligrafico e zecca dello Stato-Libreria dello Stato.

Castellani, Arrigo (1979), «Neopurismo e glottotecnica: l'intervento linguistico secondo Bruno Migliorini», in Massimo Luca Fanfani (ed.), L'opera di Bruno Migliorini nel ricordo degli allievi, con una bibliografia dei suoi scritti, Firenze, Accademia della Crusca, p. 23-39.

Colussi, Davide (2008), «Note sulla prosa del primo Migliorini», Studi linguistici italiani, n XXXIV, p. 237-259.

Cortelazzo, Manlio (2009), «Migliorini e il lessico contemporaneo», in Matteo Santipolo e Matteo Viale (ed.), Bruno Migliorini, l'uomo e il linguista (Rovigo 1896 - Firenze 1975). Atti del convegno di studi, Rovigo, Accademia dei Concordi, 11-12 aprile 2008, Rovigo, Accademia dei Concordi editore, p. 249-256.

La Crusca risponde (1995), presentazione di Giovanni Nencioni, Firenze, Le Lettere.

Della Valle, Valeria e Giuseppe Patota (2000), /l salvaitaliano, Milano, Sperling \& Kupfer Editori.

Durante, Marcello (1982), Dal latino all'italiano moderno, saggio di storia linguistica e culturale, Bologna, Zanichelli.

Fanfani, Massimo L. (2009), «La prima stagione di Lingua Nostra», in Matteo Santipolo e Matteo Viale (ed.), Bruno Migliorini, l'uomo e il linguista (Rovigo 1896 - Firenze 1975). Atti del convegno di studi, Rovigo, Accademia dei Concordi, 11 -12 aprile 2008, Rovigo, Accademia dei Concordi editore, p. 2596.

Fanfani, Pietro e Arlia, Costantino (1881), Lessico dell'infima e corrotta italianità, Milano, Carrara.

Fioritto, Alfredo (2009), Manuale di stile dei documenti amministrativi, Bologna, Il Mulino.

Folena, Gianfranco (1979), «La vocazione di Bruno Migliorini. Dal nome proprio al nome comune», in Massimo Luca Fanfani (ed.), L'opera di Bruno Migliorini nel ricordo degli allievi, con una bibliografia dei suoi scritti, Firenze, Accademia della Crusca, p. 1-16.

Franceschini, Fabrizio e Gigli, Sara (ed.) (2003), Manuale di scrittura amministrativa, Roma, s.e.

Gabrielli, Aldo (1956), Dizionario linguistico moderno. Guida pratica per scrivere e parlar bene, Milano, Mondadori.

Gabrielli, Aldo (1976), Si dice o non si dice? Aggiunte alla grammatica, Milano, Mondadori.

Gabrielli, Aldo (1977), Il museo degli errori. L'italiano come si parla oggi, Milano, Mondadori. 
Gabrielli, Aldo (1978), Nella foresta del vocabolario. Storia di parole, Milano, Mondadori.

GDLI: Grande dizionario della lingua italiana (1961-2002), 21 vol., fondato da Salvatore Battaglia, poi diretto da Giorgio Bàrberi Squarotti, Torino, Utet.

Ghinassi, Ghino (1979), «Ricordo di Bruno Migliorini dal "laboratorio" di Lingua Nostra», in Massimo L. Fanfani (ed.), L'opera di Bruno Migliorini nel ricordo degli allievi, con una bibliografia dei suoi scritti, Firenze, Accademia della Crusca, p. 41-49.

Ghinassi, Ghino (1990), «Migliorini contemporaneista», introduzione a Bruno Migliorini, La lingua italiana nel Novecento, a cura di Massimo L. Fanfani, con un saggio introduttivo di Ghino Ghinassi, Firenze, Le Lettere, p. VII-XCVI. [Riedizione di due volumi di Bruno Migliorini: Lingua contemporanea (Firenze, Sansoni, 1938) e Saggi sulla lingua del Novecento (Firenze, Sansoni, 1941).]

Migliorini, Bruno (1973), Lingua d’oggi e di ieri, Caltanissetta/Roma, Salvatore Sciascia editore.

Migliorini, Bruno (1978), Storia della lingua italiana, $2^{\text {a }}$ ed., Firenze, Sansoni. [1ª ed., 1960.]

Migliorini, Bruno (1990), La lingua italiana nel Novecento, a cura di Massimo L. Fanfani, con un saggio introduttivo di Ghino Ghinassi, Firenze, Le Lettere. [Riedizione di due volumi di Bruno Migliorini: Lingua contemporanea (Firenze, Sansoni, 1938) e Saggi sulla lingua del Novecento (Firenze, Sansoni, 1941).]

Monelli, Paolo (1943), Barbaro dominio, Milano, Hoepli.

Nencioni, Giovanni (1976), «Bruno Migliorini», Archivio Glottologico Italiano, n LXI, p. 20-36.

Nencioni, Giovanni (1997), «Plurilinguismo in Europa», La Crusca pervoi, nº 15, p. 1-4.

Panzini, Alfredo (1933), Guida alla grammatica italiana, con un prontuario delle incertezze. Libretto utile per ogni persona, Firenze, R. Bemporad \& figlio editori.

Peruzzi, Emilio (1961), Una lingua per gli italiani, Torino, ERI.

Rigutini, Giuseppe (1905), I neologismi buoni e cattivi più frequenti nell'uso odierno, Firenze, Barbera.

Serianni, Luca (1989), Grammatica italiana. Italiano comune e lingua letteraria, con la collaborazione di Alberto Castelvecchi, Torino, Utet libreria.

Serianni, Luca (2004), «Il sentimento della norma linguistica nell'Italia di oggi», Studi linguistici italiani, nºXX, p. 85-103.

Serianni, Luca (2009), «L'eredità linguistica di Bruno Migliorini: una testimonianza», in Matteo Santipolo e Matteo Viale (ed.), Bruno Migliorini, l'uomo e il linguista (Rovigo 1896 - Firenze 1975). Atti del convegno di studi, Rovigo, Accademia dei Concordi, 11-12 aprile 2008, Rovigo, Accademia dei Concordi editore, p. 9-13.

Serianni, Luca (2012), «Le riviste di storia della lingua italiana nella nascita e nello sviluppo della disciplina», Studi linguistici italiani, nº XXXVIII, p. 50-60. 
Ugolini, Filippo (1858), Vocabolario di parole e modi errati che sono comunemente in uso..., Palermo, Russo-Lao.

Viale, Matteo (2009), «Migliorini tra grammatica ed educazione linguistica», in Matteo Santipolo e Matteo Viale (ed.), Bruno Migliorini, l'uomo e il linguista (Rovigo 1896 - Firenze 1975). Atti del convegno di studi, Rovigo, Accademia dei Concordi, 11-12 aprile 2008, Rovigo, Accademia dei Concordi editore, p. 291-311. 